Kansas State University Libraries

New Prairie Press

\title{
COMPARING EXPERIMENTAL DESIGNS FOR A BI-LOGISTICAL MODEL USED TO ESTIMATE HEAT STRESS WHEN MOVING FEEDLOT CATTLE
}

X. Li

A. M. Parkhurst

T. L. Mader

Follow this and additional works at: https://newprairiepress.org/agstatconference

Part of the Agriculture Commons, and the Applied Statistics Commons

\section{(c) (1) $\Theta($}

This work is licensed under a Creative Commons Attribution-Noncommercial-No Derivative Works 4.0 License.

\section{Recommended Citation}

Li, X.; Parkhurst, A. M.; and Mader, T. L. (2009). "COMPARING EXPERIMENTAL DESIGNS FOR A BILOGISTICAL MODEL USED TO ESTIMATE HEAT STRESS WHEN MOVING FEEDLOT CATTLE," Conference on Applied Statistics in Agriculture. https://doi.org/10.4148/2475-7772.1085

This is brought to you for free and open access by the Conferences at New Prairie Press. It has been accepted for inclusion in Conference on Applied Statistics in Agriculture by an authorized administrator of New Prairie Press. For more information, please contact cads@k-state.edu. 


\title{
COMPARING EXPERIMENTAL DESIGNS FOR A BI-LOGISTICAL MODEL USED TO ESTIMATE HEAT STRESS WHEN MOVING FEEDLOT CATTLE
}

\author{
X. $\mathrm{Li}^{1}$, A. M. Parkhurst ${ }^{1}$, and T. L. Mader ${ }^{2}$ \\ ${ }^{1}$ Department of Statistics, University of Nebraska at Lincoln \\ ${ }^{2}$ Department of Animal Science, University of Nebraska at Lincoln
}

\begin{abstract}
Processing and handling cattle requires expenditure of energy causing an elevation of body temperature, depending on the ambient conditions. Therefore, caution should be exercised in moving cattle, especially during summer. More knowledge of the dynamics of body temperature, $(\mathrm{Tb})$, could lead to specific recommendations on how far and under what conditions cattle can be moved before becoming thermally challenged. Data comes from feedlot trials conducted over four days. A bi-logistic mixed model of $\mathrm{Tb}$ is used to describe the effects of moving and handling on $\mathrm{Tb}$. This model provides estimates for several important biological parameters describing the thermal challenge and recovery: the maximum $\mathrm{Tb}$ challenge, challenge rate constant (rate constant for increase in $\mathrm{Tb}$ ), time to maximum rate of challenge (challenge inflection point), baseline for recovery, recovery rate constant (rate constant for decrease in $\mathrm{Tb}$ ) and time to maximum recovery rate (recovery inflection point). Fitting a nonlinear mixed model with six parameters under extremely variable animal and environmental conditions is difficult especially when the treatment factor (distance) is introduced into the model. Additional difficulties in fitting the model arise as the experimental design increases in complexity from a CRD to a replicated Latin square. The objectives of this study are: to examine the bi-logistic model with distance as a treatment factor and estimate the relative efficiencies as the experimental design is simplified.
\end{abstract}

\section{INTRODUCTION}

There are over 10 million head of cattle feed in feedlots in the Great Plains and Western Cornbelt of the United States at any one time. Processes, such as vaccination and treatment for parasites, are done for cattle within a few days after they came into the feedlot. In most cases, cattle are returned to the processing facilities to receive health care and all cattle have to be moved or handled when they are shipped to the packing plant once they are finished. Both processing and handling of cattle can cause a sharp rise in body temperature, especially during hot ambient conditions and this increases the risk of heat stress in cattle. (Parkhurst and Mader, 2000)

Heat stress affects animals' growth and results in reduced feed intake. Heat stress also causes economic losses to livestock producers. In extreme cases, heat stress can even result in death of susceptible cattle. For example, the heat waves in Iowa (1995) and Nebraska (1999) resulted in death lost of more than 3,500 head of cattle and million dollar losses in the beef industry. The heat wave in California (July 2006) resulted in over \$1 billion losses and higher food prices due to a lower production (Aitha et al. 2007).

Previous work by Parkhurst and Mader(2000) suggests using the nonlinear bi-logistic model to describe the steers' movement and handling during the summer. Our focus is on modeling the 
growth and decay of an animal's body temperature during a moving event in order to characterize the dynamics of the moving process and additionally provide animal-level prediction. It is a subject-specific model which explicitly acknowledges individual behavior while incorporating scientific characterizations in the expectation function. The nonlinear mixed-effect formulation allows for considerable flexibility in the specification of the random-effects structure and can be extended to allow for heteroscedastic and correlated within-groups errors. Thus, we use the nonlinear bi-logistic model to fit data from an experiment conducted as a replicated Latin square with two distance treatments. However, nonlinear analysis is very sensitive to the choice of starting values. Thus, the analysis begins with the simplest experimental design, a completely randomized design (CRD). We then progress to a crossover design using pen as a crossover factor. Then, we analyze the replicated Latin square design. Finally, we compare the relative efficiencies of all three designs.

Thus, in this study, there are three objectives.

1. Investigate use of bi-logistic model to describe the moving event and estimate the parameters.

2. Determine significance of heat stress by comparing effects of distance (200 meters vs. 1200 meters) as body temperature responds to heat challenge and recovery during moving event.

3. Compare analyses as experimental design increases in complexity from a completely randomized design to a crossover design to a replicated Latin square based on interpretation of parameter estimates, information criteria and residual diagnostics.

\section{MATERIALS AND METHODS}

\section{2.a Data and Experimental Design}

The data were taken every two minutes from Aug. $10^{\text {th }}$ to Aug. $14^{\text {th }}$ in 1999 . The body temperature $(\mathrm{Tb})$ of the steers was measured as the ear's tympanic temperature. Four steers were chosen. Two of them were assigned to pen 1 and the other two were assigned to pen 2 . The pens were identical in size and bunk space. On Aug. $9^{\text {th }}$, all four steers were weighed and tympanic data-loggers were placed in the left ear to record body temperature at 2-minute intervals. The experiment was conducted as a replicated Latin square, Table 2.1. On Aug. $10^{\text {th }}$ and $11^{\text {th }}$ (period 1), the steers in pen 1 were moved approximately 200 meters (treatment level 1). The steers in pen 2 were moved approximately 1200 meters (treatment level 2). The four steers were not moved on Aug. $12^{\text {th }}$. On Aug. $13^{\text {th }}$ and $14^{\text {th }}$, the steers in each pen were assigned the opposite treatment and moved accordingly.

Table 2.1 Replicated Latin Square Design for Experiment

\begin{tabular}{|c|c|c|c|c|c|c|}
\hline Pen & Steer & $\begin{array}{l}\text { Aug } 10 \\
\text { Day } 1\end{array}$ & $\begin{array}{l}\text { Aug } 11 \\
\text { Day } 2\end{array}$ & & $\begin{array}{c}\text { Aug } 13 \\
\text { Day } 3\end{array}$ & $\begin{array}{r}\text { Aug } 14 \\
\text { Day } 4\end{array}$ \\
\hline & 377 & 200 & 200 & \multirow{4}{*}{$\stackrel{3}{\simeq}$} & 1200 & 1200 \\
\hline & 445 & 200 & 200 & & 1200 & 1200 \\
\hline \multirow[t]{2}{*}{2} & 442 & 1200 & 1200 & & 200 & 200 \\
\hline & 508 & 1200 & 1200 & & 200 & 200 \\
\hline
\end{tabular}




\section{Figure 1. Observed Tympanic Temperature versus Time for each Pen-Steer/Day}

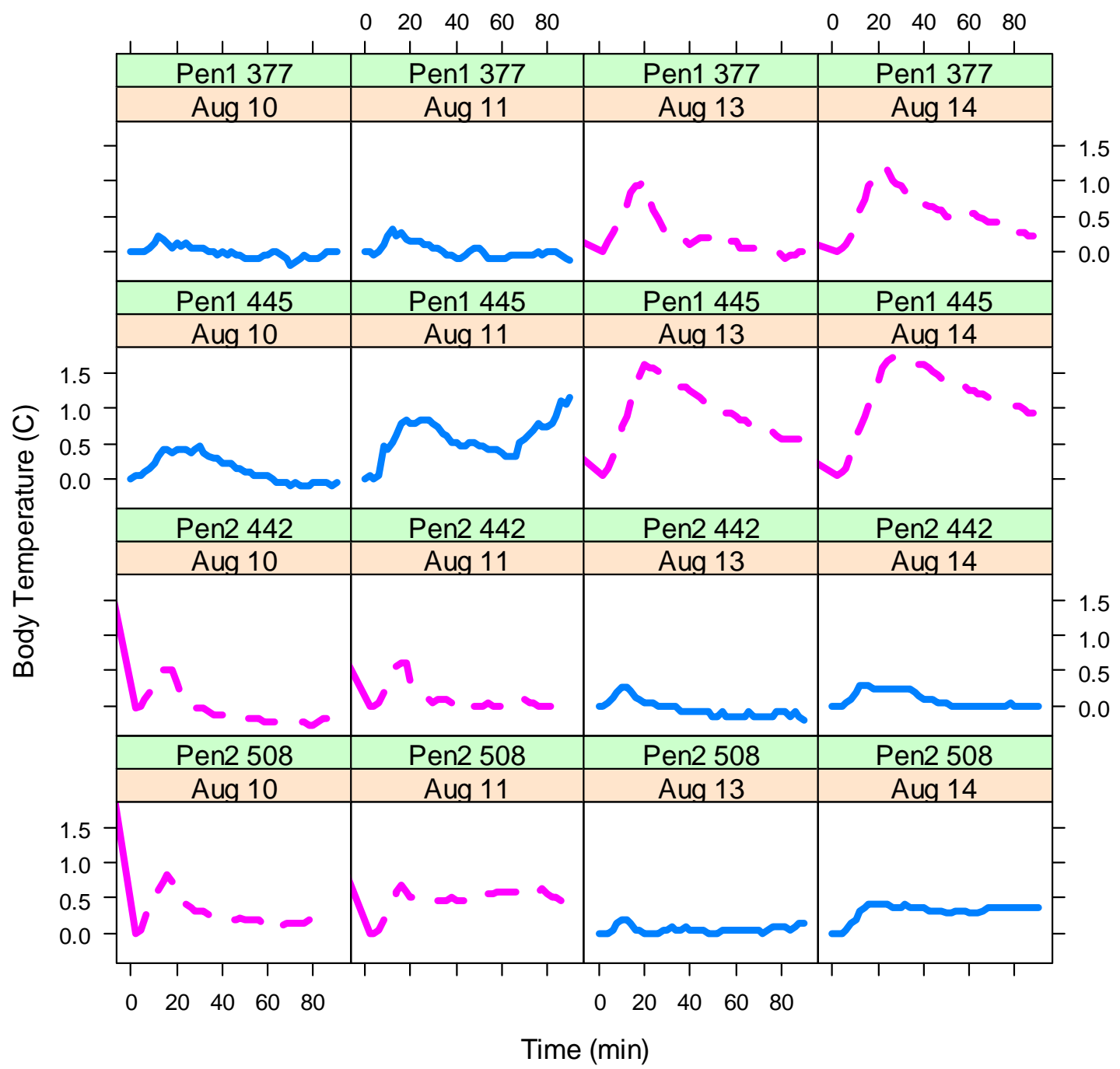

200 meter run

1200 meter run

Figure 1 shows the raw data for each steer in each pen over the four runs on Aug 10 to Aug 14. Data is not shown for Aug 12 since there were no runs. The short 200 meter runs are indicated by a solid line; the long 1200 meter runs by a dashed line. From the plot, we note the following points:

1. For each run, there is a sharp rise in $\mathrm{Tb}$.

2. Tb tends to return to baseline of zero.

3. The peaks of the long runs are higher than those of the short runs.

From these observations and prior research (Parkhurst and Mader, 2000), we choose the sixparameter bi-logistic model to analysis this dataset. 


\section{2.b Expectation function}

The six-parameter bi-logistic model results in the expectation function given by

$$
y=\frac{\alpha}{1+e^{\kappa_{1}\left(X-\tau_{1}\right)}}+\frac{\delta-\alpha}{1+e^{\kappa_{2}\left(X-\tau_{2}\right)}} \quad \text { Eq1 }
$$

Where the response variable, $\mathrm{y}$, is the body temperature of the steers which is measured every 2 minutes and the independent variable, $\mathrm{X}$, is the time (minutes) at which the steers' body temperature is taken. There are six parameters in the model: $\alpha$ is the maximum $\mathrm{Tb}, \kappa_{1}$ is the challenge rate constant, $\tau_{1}$ is challenge inflection point, $\delta$ is the baseline for recovery, $\kappa_{2}$ is the rate constant for heat recovery, and $\tau_{2}$ is challenge inflection point.

\section{2.c Random Effects Structures for Three Experimental Designs}

\section{2.c.i Completely Randomized Design}

Besides the fixed effect parameters specified above, the random effects are also considered. For each of the four days, there are two pens and two steers within each pen. Thus, we consider the pen and the steers within pen as hierarchal random effects. The variation in days is included in the error term. The factor in the experiment is the distance.

The fixed and random effects for each of the six parameters are

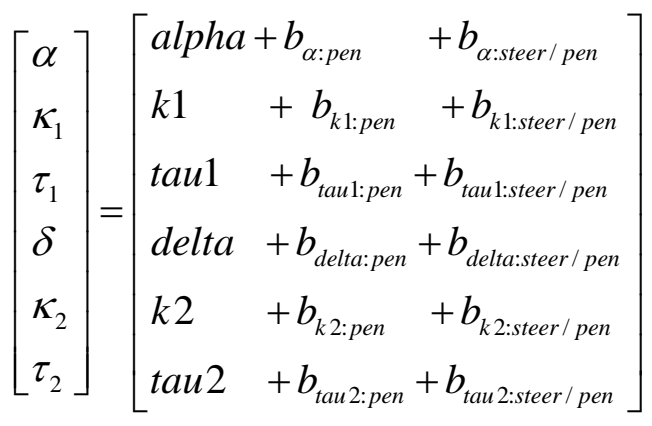

\section{Eq 2}

Both fixed and random effects are considered for the six parameters: $\alpha, \kappa_{1}, \tau_{1}, \delta, \kappa_{2}$ and $\tau_{2}$. The fixed effects, alpha, k1, taul, delta, $k 2$ and tau 2 represent population means. Random effects $\mathbf{b}_{\text {pen }}=\left[b_{\alpha: p e n}, b_{k 1: p e n}, b_{\text {taul:pen }}, b_{\text {delta:pen }}, b_{k 2: p e n}\right.$ and $\left.b_{\text {tau2:pen }}\right]$ represent the deviation from the population means associated with each pen. Random effects $\mathbf{b}_{\text {steer/pen }}=\left[b_{\alpha: s t e e r / p e n}, b_{k 1: \text { steer } / p e n}\right.$, $b_{\text {taul:ster/ pen }}, b_{\text {deltaster/pen }}, b_{k 2 \text { :steer/pen }}$ and $b_{\text {tau2:steer/pen }}$ ] represent the deviations from the population means associated with each steer within a pen We assume that random effects are independent; that is, $\mathbf{b}_{\text {pen }} \sim \mathbf{N}\left(\mathbf{0}, \psi_{\text {pen }}\right), \mathbf{b}_{\text {steer/pen }} \sim \mathbf{N}\left(\mathbf{0}, \psi_{\text {steer/pen }}\right)$, and $\boldsymbol{\varepsilon} \sim \mathbf{N}\left(\mathbf{0}, \boldsymbol{\sigma}^{2}\right.$ I) are independent of each other. 
The covariance for pens, $\Psi_{p e n}$, is a diagonal matrix.

$$
\Psi_{\text {pen }} \sim\left(\begin{array}{cccc}
\sigma_{\text {alphapen }}^{2} & 0 & 0 & 0 \\
& \sigma_{k 1: p e n}^{2} & 0 & 0 \\
& & \ddots & 0 \\
& & & \sigma_{\text {tau2:pen }}^{2}
\end{array}\right)
$$

Eq 3

The steers within pen covariance matrix $\Psi_{\text {steer/pen }}$, is the diagonal matrix.

$$
\Psi_{\text {ster/pen }} \sim\left(\begin{array}{cccc}
\sigma_{\text {alphasteer/pen }}^{2} & 0 & 0 & 0 \\
& \sigma_{\text {k1:steer/pen }}^{2} & 0 & 0 \\
& & \ddots & 0 \\
& & & \sigma_{\text {tau2:steer/pen }}^{2}
\end{array}\right) \quad \mathrm{Eq} 4
$$

When distance is included as a treatment factor, the expectation function for CRD is given by the following modification of Equation 1.

$$
\begin{aligned}
& y=\frac{\alpha}{1+e^{\kappa_{1}\left(X-\tau_{1}\right)}}+\frac{\delta-\alpha}{1+e^{\kappa_{2}\left(X-\tau_{2}\right)}} \\
& {\left[\begin{array}{l}
\alpha \\
\kappa_{1} \\
\tau_{1} \\
\delta \\
\kappa_{2} \\
\tau_{2}
\end{array}\right]=\left[\begin{array}{ll}
\text { alpha }+D C+b_{\alpha: p e n}+b_{\alpha: \text { steer } / p e n} \\
k 1+D C+b_{k 1: p e n}+b_{k 1: \text { steer } / p e n} \\
\text { tau } 1+D C+b_{\text {taul:pen }}+b_{\text {tau1:ster } / p e n} \\
\text { delta }+D C+b_{\text {delta:pen }}+b_{\text {delta:steer } / p e n} \\
\text { tau2 }+D C+b_{k 2: p e n}+b_{k 2: s t e e r / p e n}
\end{array}\right.} \\
& C=\left\{\begin{array}{l}
0, \text { for } 200 \text { meter run } \\
1, \text { for } 1200 \text { meter run }
\end{array}\right.
\end{aligned}
$$

And D represents the coefficients of the distance treatment effects.

Table 2.2 R-code for nlme with CRD

strdata.allday.group<-groupedData(ytb time $\mid$ pen/anm, data=strdata.allday, order.groups=TRUE)

steers.all.nlme.trt<-nlme $(\mathrm{ytb} \sim \mathrm{alpha} /(1+\exp (\mathrm{k} 1 *($ time$-\mathrm{tau} 1)))+($ deltaalpha $) /(1+\exp (\mathrm{k} 2 *($ time-tau 2$)))$,

fixed $=$ alpha $+\mathrm{k} 1+$ tau $1+$ delta $+\mathrm{k} 2+$ tau2 factor(trt), random $=$ pdDiag $($ alpha $+\mathrm{k} 1+$ tau $1+$ delta $+\mathrm{k} 2+$ tau2 1$)$, data $=$ strdata.allday.group, control $=$ list $\left(\right.$ maxiter $=100000$, minFactor $\left.=0.5^{\wedge} 2048\right)$, start $=\mathrm{c}(1.04667,3,-0.29804,0,10.24813,4$, $0.10620,3,-0.09614,0,26.30476,5))$ 


\section{2.c.ii Crossover Design}

For this design, the steers serve as their own control to compare the two distance treatments: S (short, 200 meters, $\mathrm{C}=0$ ) and $\mathrm{L}$ (long, 1200 meters, $\mathrm{C}=1$ ) in a $2 \times 2$ crossover design. The steers within a pen are randomized to one of the treatment sequences - either SL or LS. Steers within a pen randomized to the SL sequence run the short distance first then "cross over" and run the long distance. Between the two treatments, a day of no runs provides a "wash out" period. The treatment is applied to the pen for two days. There are two steers in each pen for each day. We treat pen as a crossover factor. Pen, day and steers within pen-day are considered as random effects.

The fixed and random effects for the six parameters are:

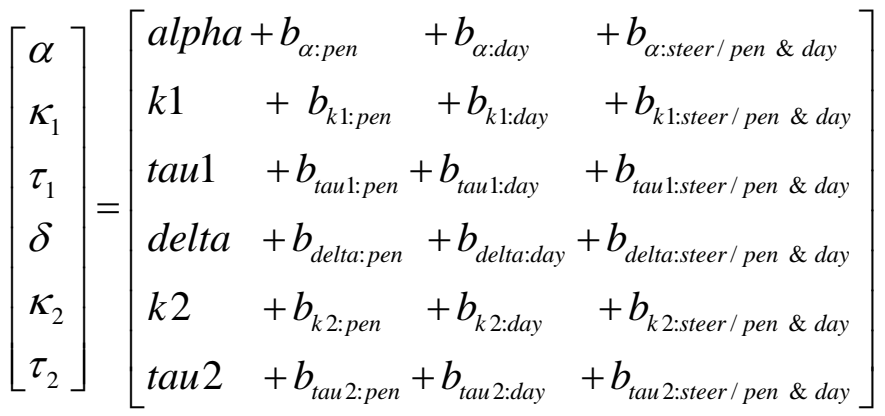

Both fixed and random effects are considered for the six parameters: $\alpha, \kappa_{1}, \tau_{1}, \delta, \kappa_{2}$ and $\tau_{2}$. The fixed effects, alpha, $k 1$, taul, delta, $k 2$ and tau 2 represent population means. Random effects $\mathbf{b}_{\text {pen }}=\left[b_{\alpha: p e n}, b_{k 1: p e n}, b_{\text {taul:pen }}, b_{\text {delta:pen }}, b_{k 2: p e n}\right.$ and $\left.b_{\text {tau2:pen }}\right]$ represents the deviation from the population means associated with each pen. Random effects $\mathbf{b}_{\text {day }}=\left[b_{\alpha: d a y}, b_{k 1: \text { day }}, b_{\text {taul:day }}, b_{\text {delta:day }}\right.$, $b_{k 2: d a y}$ and $\left.b_{\text {tau2:day }}\right]$ represent the deviation from the population means associated with each day. Random effects $\mathbf{b}_{\text {steer/pen\&day }}=\left[b_{\alpha: \text { steer } / \text { pen\&day }}, b_{k 1: \text { steer/pen\&day }}, b_{\text {taul:steer } / \text { pen\&day }}, b_{\text {deltasteer } / \text { pen\&day }}\right.$, $b_{k 2 \text { :steer/pen\&day }}$ and $b_{\text {tau2:steer/ pen\&day }}$ ] represent the deviations from the population means associated with each steer within a pen-day combination. We assume that random effects are independent; that is, $\mathbf{b}_{\text {pen }} \sim \mathbf{N}\left(\mathbf{0}, \boldsymbol{\psi}_{\text {pen }}\right), \mathbf{b}_{\text {day }} \sim \mathbf{N}\left(\mathbf{0}, \sigma_{\text {day }}^{2} I\right), \mathbf{b}_{\text {steer/pen\&day }} \sim \mathbf{N}\left(\mathbf{0}, \boldsymbol{\psi}_{\text {steer/pen\&day }}\right)$, and $\boldsymbol{\varepsilon} \sim \mathbf{N}\left(\mathbf{0}, \boldsymbol{\sigma}^{2} I\right)$ are independent each other.

The covariance for pens, $\Psi_{p e n}$, is a diagonal matrix.

$$
\Psi_{p e n} \sim\left(\begin{array}{cccc}
\sigma_{\text {alphapen }}^{2} & 0 & 0 & 0 \\
& \sigma_{k 1: p e n}^{2} & 0 & 0 \\
& & \ddots & 0 \\
& & & \sigma_{\text {tau2:pen }}^{2}
\end{array}\right)
$$


The steers within pen and day covariance matrix $\varepsilon_{\text {steer/pen\&day }}$, is diagonal matrix.

$$
\Psi_{\text {steer/pen \& day }} \sim\left(\begin{array}{cccc}
\sigma_{\text {alphasteer/ pen\&day }}^{2} & 0 & 0 & 0 \\
& \sigma_{k 1 \text { steer/pen\&day }}^{2} & 0 & 0 \\
& & \ddots & 0 \\
& & & \sigma_{\text {tau2:ster/ pen\&day }}^{2}
\end{array}\right)
$$

Eq 8

When distance is included as a treatment factor, the expectation function for Crossover design is given by the following modification of Equation 1 .

$$
y=\frac{\alpha}{1+e^{\kappa_{1}\left(X-\tau_{1}\right)}}+\frac{\delta-\alpha}{1+e^{\kappa_{2}\left(X-\tau_{2}\right)}}
$$

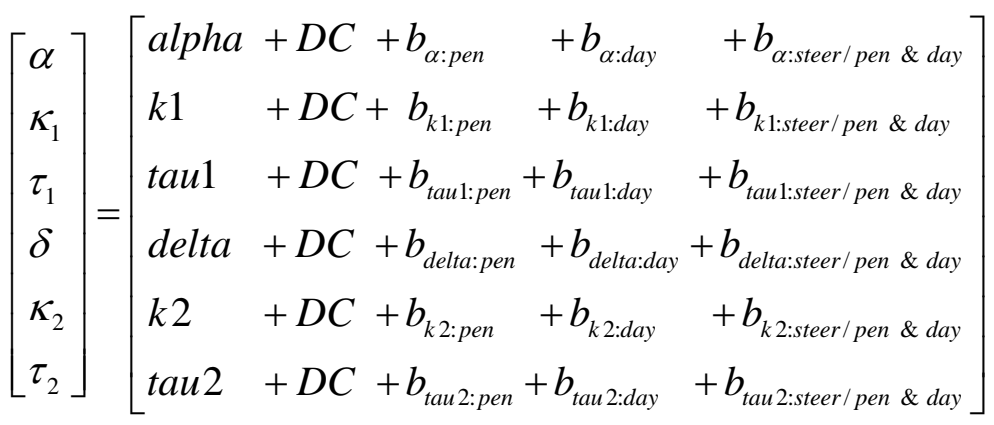

$C= \begin{cases}0, & \text { for } 200 \text { meter run } \\ 1, & \text { for } 1200 \text { meter run }\end{cases}$

And D represents the coefficients of the distance treatment effects.

Table 2.3 R-code for nlme with Crossover Design (Zhou et al, 2007) steers.crossover.trt<-nlme $(\mathrm{ytb}$ alpha/ $(1+\exp (\mathrm{k} 1 *($ time-tau 1$)))+($ deltaalpha $) /(1+\exp (\mathrm{k} 2 *($ time-tau 2$)))$,

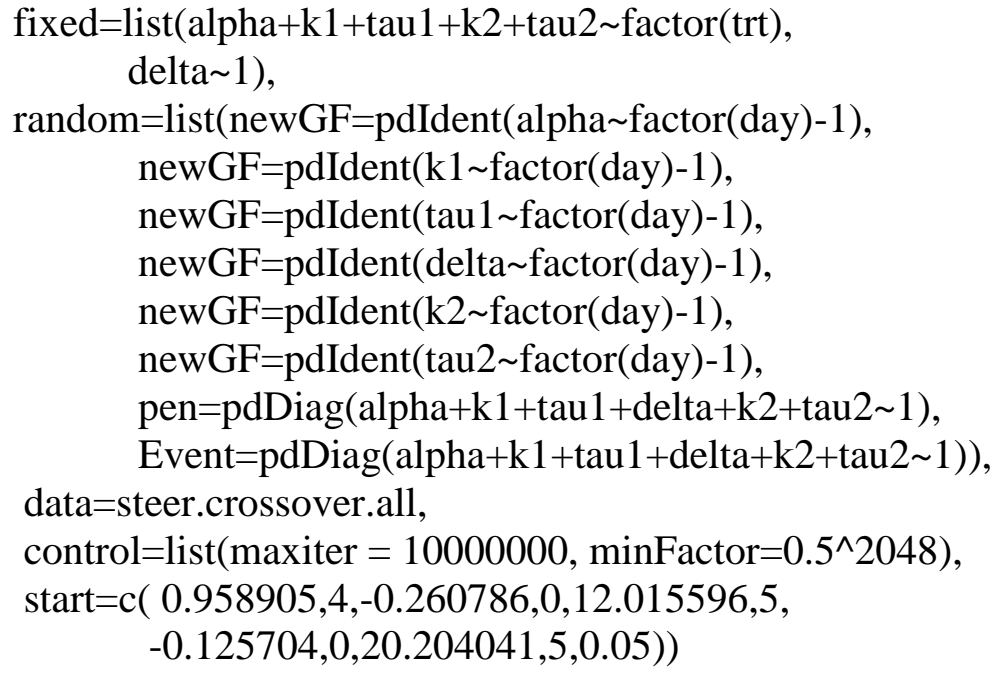




\section{2.c.iii Replicated Latin Square}

There are two squares. The first days, one and three, are considered as the first Latin square; the second days, two and four, are consider as the second Latin square. Thus, there are two 2x2 Latin squares. Pens are the row effect; day is the column effect and for the first square the focus is in the initial day of the run. The two treatment distances (1200 meters and 200 meters) are randomly assigned to occur once in each row (pen) and once in each column (initial day). The second square, which focuses on the second day of the run, is similar. Since there are two steers in each pen for each day, the pen-day combination may be considered an event. We consider square, pen within square, day within square, and steer within pen-day within square as random effects.

The fixed and random effects for each of the six parameters are

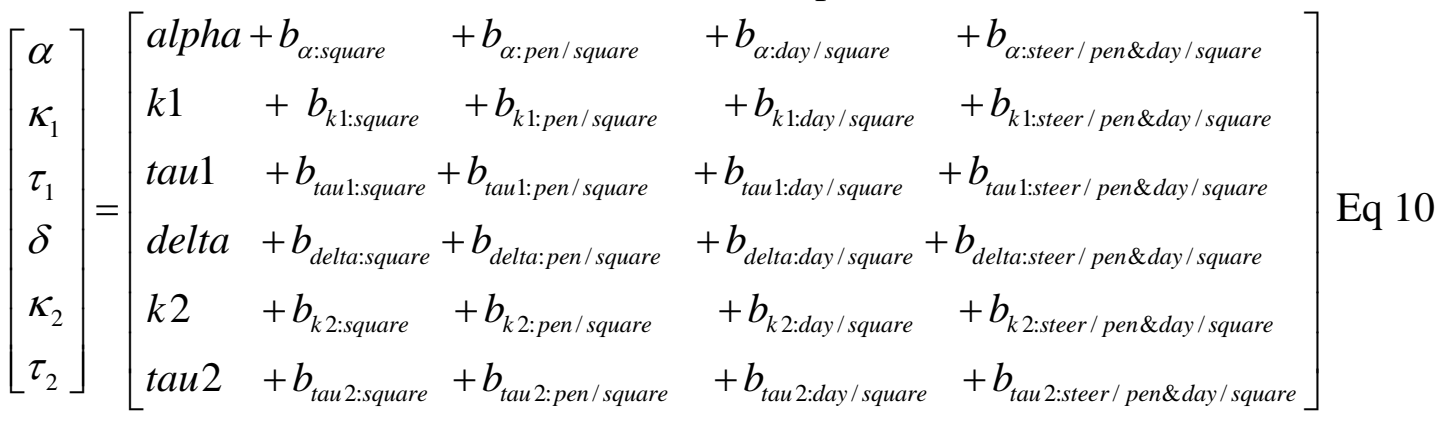

Both fixed and random effects are considered for the six parameters $\alpha, \kappa_{1}, \tau_{1}, \delta, \kappa_{2}$ and $\tau_{2}$. The fixed effects, alpha, $k 1$, taul, delta, $k 2$ and tau 2 represent population means. Random effects $\mathbf{b}_{\text {square }}=\left[b_{\alpha: s q u a r e}, b_{k 1: s q u a r e}, b_{\text {taul:square }}, b_{\text {deltasquare }}, b_{k 2: s q u a r e}\right.$ and $\left.b_{\text {tau2:square }}\right]$ represent the deviation from the population means associated with each square. Random effects $\mathbf{b}_{\text {pen/square }}=\left[b_{\alpha: \text { pen/square }}\right.$, $b_{k 1: p e n / s q u a r e}, b_{\text {taul:pen/square }}, b_{\text {delta:pen/square }}, b_{k 2: \text { pen/square }}$ and $\left.b_{\text {tau2:pen/square }}\right]$ represent the deviation from the population means associated with each pen within a square. Random effects $\mathbf{b}_{\text {day/square }}=[$ $b_{\alpha: d a y / s q u a r e}, b_{k 1: d a y / s q u a r e}, b_{\text {taul:day/square }}, b_{\text {deltadday/square }}, b_{k 2: d a y / s q u a r e}$ and $\left.b_{\text {tau2:day/square }}\right]$ represent the deviation from the population means associated with each day within a square. Random effects

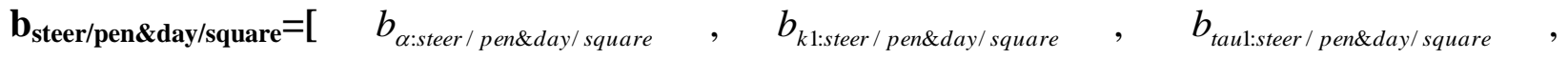
$b_{\text {deltasteer/pen\&day/square }}, b_{k 2 \text { :steer/pen\&day/square }}$ and $b_{\text {tau2:steer/pen\&day/square }}$ represent the deviations from the population means associated with each steer within a pen and a day within a square. We assume that random effects are independent; that is, $\mathbf{b}_{\text {square }}{ }^{\sim} \mathbf{N}\left(\mathbf{0}, \sigma_{\text {sqaure }}^{2}\right)$, $\mathbf{b}_{\text {pen/square }} \sim \mathbf{N}\left(\mathbf{0}, \boldsymbol{\psi}_{\text {pen/square }}\right), \mathbf{b}_{\text {day/square }} \sim \mathbf{N}\left(\mathbf{0}, \sigma_{\text {day/square }}^{2} I\right), \mathbf{b}_{\text {steer/pen } \& \text { day/square }} \sim \mathbf{N}\left(\mathbf{0}, \psi_{\text {steer/pen } \& \text { day/square }}\right)$, and $\boldsymbol{\varepsilon} \sim \mathbf{N}\left(\mathbf{0}, \boldsymbol{\sigma}^{2} \mathrm{I}\right)$ are independent of each other. 
The covariance for pens within square, $\Psi_{\text {pen/square }}$, assumed to be a diagonal matrix.

$$
\Psi_{\text {pen/square }} \sim\left(\begin{array}{cccc}
\sigma_{\text {alphapen/square }}^{2} & 0 & 0 & 0 \\
& \sigma_{k 1: p e n / s q u a r e}^{2} & 0 & 0 \\
& & \ddots & 0 \\
& & & \sigma_{\text {tau2:pen/square }}^{2}
\end{array}\right)
$$

Eq 11

The covariance for steers within pen and day within square, $\Psi_{\text {steer/pen\&day/square }}$ is a diagonal matrix.

$\Psi_{\text {steer/pen\&day/square }} \sim\left(\begin{array}{cccc}\sigma_{\text {alphasteer/pen\&day/square }}^{2} & 0 & 0 & 0 \\ & \sigma_{k 1: s t e e r / p e n \& d a y / s q u a r e}^{2} & 0 & 0 \\ & & \ddots & 0 \\ & & & \sigma_{\text {tau2:steer/pen\&day/square }}^{2}\end{array}\right)$ Eq 12

When distance is included as a treatment factor, the expectation function for replicated Latin square design is given by the following modification of Equation 1.

$$
\begin{aligned}
& y=\frac{\alpha}{1+e^{\kappa_{1}\left(X-\tau_{1}\right)}}+\frac{\delta-\alpha}{1+e^{\kappa_{2}\left(X-\tau_{2}\right)}}
\end{aligned}
$$

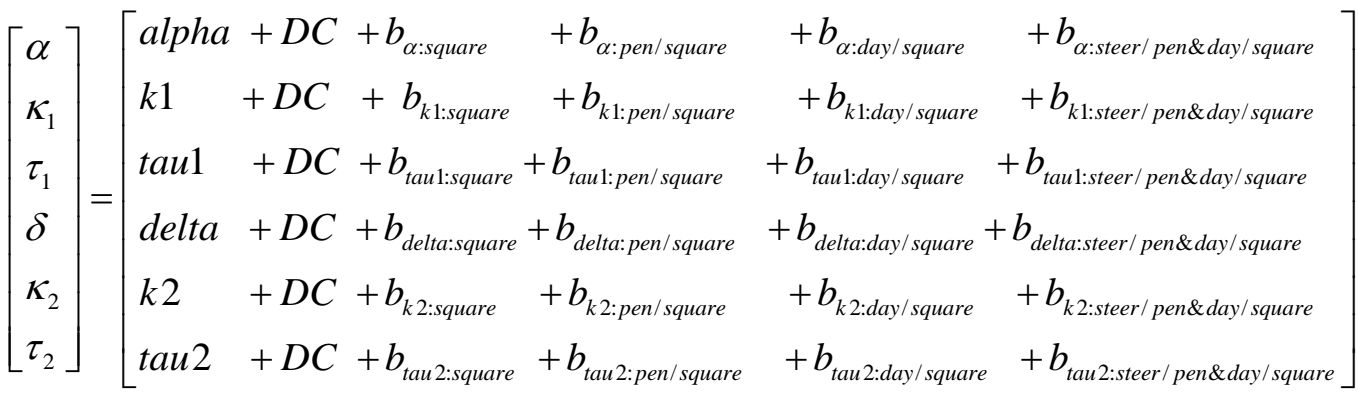

$$
\begin{aligned}
& C= \begin{cases}0, & \text { for } 200 \text { meter run } \\
1, & \text { for } 1200 \text { meter run }\end{cases}
\end{aligned}
$$

And D represents the coefficients of the distance treatment effects. 
Table 2.d.3 R-CODE for nlme with replicated Latin square design (Zhou et al, 2006)

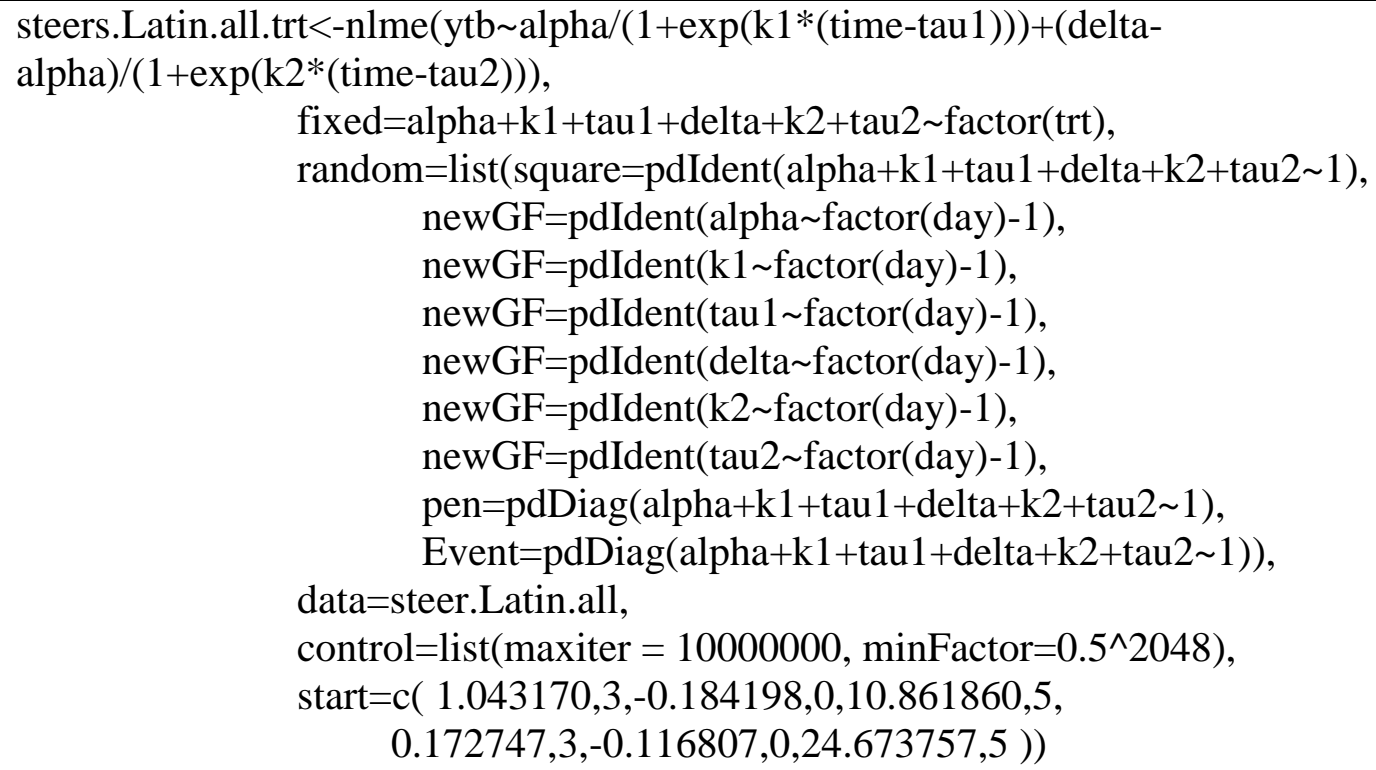

\section{2.d Model Building}

\section{2.d.i Fixed effects}

All analyses are performed in $\mathbf{R}$ version 2.8.0. Initially we fit the model with only fixed effects but no random effects using $n l s$ procedure. In this way, we estimate parameters and check their information provided in the raw data graph. The parameter estimates provide possible starting values for the mixed models. For the mixed model, the data are grouped according to the experimental design. The mixed model is fit using nlme and compared to the fixed model to test the importance of using random effects.

\section{2.d.ii Random Effects}

To identify which random effects are required, we fit the nonlinear mixed model without the treatment effect for several sets of random effects and check for model equivalency. We begin with the full model. Then, we remove the random effect which has the smallest estimated standard deviation and compare the models to see if the removed random effect is necessary. We use the likelihood ratio test to test for non-significance, $p>0.25$. If the new model is nonsignificant, we select that model and repeat the process until the next model is significantly different.

\section{2.d.iii Including Treatment Factors}

After fitting the fixed effects and random effects, we take the treatment effect of distance into account. We compare mixed model analysis with and without the distance using information criteria and the likelihood ratio test. The NLME with distance model fits better if it has a smaller AIC, BIC and larger log likelihood than the mixed model without the treatment effect. If the pvalue for the likelihood ratio test is $<0.0001$, the NLME with distance as a factor fits the data better than NLME without the factor, 


\section{2.e Diagnostics for each Model}

After the model converges, the residuals are analyzed for normality and independence. To check for normality we examine the Q-Q plot to see if residuals fall approximately on a straight line. The residual-time plot is a check for outliers and patterns that might indicate lack of independence. We use the code 'correlation=corAR1()' in nlme to estimate autocorrelation among residuals. We then compare models with and without ARMA, using the information criteria and the likelihood ratio test.

\section{2.f Relative Efficiencies}

Relative Efficiency of two models is the ratio their mean square errors. It is a common statistical method used to compare two models. From the relative efficiency, the better model needs fewer observations to achieve the same level of precision as the other model. We calculate the relative efficiency as

$$
R E=\frac{\text { Mean square error of model } 1}{\text { Mean square error of model } 2}
$$

\section{RESULTS AND DISCUSSION}

\section{3.a Random effects}

We begin by comparing the random effects from each design. For each design we note the magnitude of the residual error and how the variation is attributed to each parameter for each level of variation.

First, we present the results of the CRD design using the full diagonal random-effects structure for both the pen level and the steers within pen level. The estimated standard deviations of the random effects are given in Table 3.1. No violations were detected when the residuals were checked for independence and normality.

Table 3.1. Estimated Standard Deviations of Random effects for CRD

\begin{tabular}{|c|c|c|c|c|c|c|}
\hline & \multicolumn{7}{|c|}{ Non-Linear Mixed Models } \\
\hline $\begin{array}{c}\text { Random } \\
\text { Effect }\end{array}$ & alpha & $\mathbf{k} 1$ & tau1 & delta & $\mathbf{k} 2$ & tau2 \\
\hline level & Std Dev & Std Dev & Std Dev & Std Dev & Std Dev & Std Dev \\
\hline Pen & $\mathbf{3 . 1 2 5 e - 0 5}$ & $\mathbf{0 . 0 7 8 6}$ & $\mathbf{0 . 0 1 7 6}$ & $\mathbf{6 . 2 8 3 e - 0 5}$ & $\mathbf{2 . 9 3 7 e - 0 5}$ & $\mathbf{0 . 0 0 3 1 3}$ \\
\hline $\begin{array}{c}\text { Steers } \\
\text { within pen }\end{array}$ & $7.357 \mathrm{e}-07$ & 0.0175 & 2.551 & 0.154 & $1.285 \mathrm{e}-08$ & 13.599 \\
\hline Residual & \multicolumn{7}{|c|}{0.180} \\
\hline
\end{tabular}

Next, we present the results of the Crossover design using the identity random-effect structure for day level and the diagonal random-effects structure for both pen and the steers within pen level. The estimated standard deviations of the random effects are given in Table 3.2. The crossover model did not converge when treatment effects were assigned for all parameters. When the distance effect was removed from the recovery baseline, delta, the model converged and no violations were detected when the residuals were checked for independence and normality. 
Table 3.2. Estimated Standard Deviations of Random effects for CROSSOVER Design

\begin{tabular}{|c|c|c|c|c|c|c|}
\hline \multirow{2}{*}{ Random Effect } & \multicolumn{7}{|c|}{ Non-Linear Mixed Models } \\
\cline { 2 - 7 } & alpha & k1 & tau1 & delta & k2 & tau2 \\
\hline level & Std Dev & Std Dev & Std Dev & Std Dev & Std Dev & Std Dev \\
\hline $\begin{array}{c}\text { Dayi, } \\
\text { i } 1,2,3,4\end{array}$ & $1.133 \mathrm{e}-38$ & $1.687 \mathrm{e}-06$ & 1.096 & 0.00181 & $2.809 \mathrm{e}-05$ & 0.0117 \\
\hline pen & 0.000598 & $2.629 \mathrm{e}-05$ & 0.000454 & $1.287 \mathrm{e}-21$ & $2.033 \mathrm{e}-05$ & 0.0100 \\
\hline $\begin{array}{c}\text { Steers within } \\
\text { pen-day }\end{array}$ & 0.157 & $2.944 \mathrm{e}-08$ & $1.792 \mathrm{e}-05$ & 0.295 & 0.0912 & 13.185 \\
\hline Residual & & \multicolumn{7}{|c|}{0.0673} \\
\hline
\end{tabular}

Finally, we present the results of the replicated Latin square design using the identity randomeffect structure both for the square and day within square level. The diagonal random-effects structure is used in both the pen within square and the steers within pen-day within square level. The estimated standard deviations of the random effects are given in Table 3.3 The replicated Latin square design has the smallest residual which is not surprising since it accounts for more known sources of variation. No violations were detected when the residuals were checked for independence and normality.

Table 3.3. Estimated Standard Deviations of Random effects for Replicated Latin Square Design

\begin{tabular}{|c|c|c|c|c|c|c|}
\hline \multirow{2}{*}{ Random Effect } & \multicolumn{6}{|c|}{ Non-Linear Mixed Models } \\
\cline { 2 - 7 } & alpha & k1 & tau1 & delta & k2 & tau2 \\
\hline level & Std Dev & Std Dev & Std Dev & Std Dev & Std Dev & Std Dev \\
\hline Square & $1.835 \mathrm{e}-10$ & $1.835 \mathrm{e}-10$ & $1.835 \mathrm{e}-10$ & $1.835 \mathrm{e}-10$ & $1.835 \mathrm{e}-10$ & $1.835 \mathrm{e}-10$ \\
\hline $\begin{array}{c}\text { Day within } \\
\text { square } \\
\text { i=1,2 }\end{array}$ & 0.0638 & $7.073 \mathrm{e}-09$ & $1.336 \mathrm{e}-16$ & $6.558 \mathrm{e}-05$ & $5.433 \mathrm{e}-05$ & $3.569 \mathrm{e}-05$ \\
\hline $\begin{array}{c}\text { Pen within } \\
\text { square }\end{array}$ & $9.393 \mathrm{e}-06$ & $8.193 \mathrm{e}-10$ & 0.518 & 0.000509 & $6.286 \mathrm{e}-05$ & $1.282 \mathrm{e}-05$ \\
\hline $\begin{array}{c}\text { Steers within } \\
\text { pen-day within } \\
\text { square }\end{array}$ & 0.0896 & $1.264 \mathrm{e}-08$ & 1.030 & 0.374 & 0.0672 & 18.858 \\
\hline Residual & \multicolumn{7}{|c|}{0.0589} & \\
\hline
\end{tabular}

At the steer level, the standard deviations for tau1 and tau2 are large in CRD; the standard deviation for tau 2 is large in crossover design; the standard deviations for tau1 and tau2 are large in replicated Latin square design. Comparing these results, we see the standard deviations for taul are different among these three designs. In the crossover design, the standard deviation for taul is small; and in other two designs, it's large. This indicates substantial variation among steers for the recovery inflection point in the CRD and Latin square. 
For pen and day, there is no clear pattern among the designs. At the pen level, all standard deviations are small; the largest standard deviation is for tau1 in replicated Latin square design. At the day level, the standard deviation for tau1 is large in the crossover design; the standard deviation for alpha is large in the replicated Latin square design compared to the crossover design.

At the square level, all standard deviations are small in the replicated Latin square design. The variation between the two squares appears negligible.

\section{3.b Fixed effects}

Table 3.4 summarizes the fixed effect estimates for each parameter distance effect for each experimental design. The short distance $(200 \mathrm{~m})$ is considered to be the reference treatment and the long distance $(1200 \mathrm{~m})$ is the additional effect. For instance, alpha, the maximum increase in $\mathrm{Tb}$ for $200 \mathrm{~m}$ in CRD is 0.61 . The maximum increase for $\mathrm{Tb}$ for $1200 \mathrm{~m}$ in CRD is $0.61+1.61=2.22 \mathrm{C}$.

Table 3.4. Estimation of fixed effects for all designs

\begin{tabular}{|l|c|l|l|l|l|l|}
\hline & \multicolumn{2}{|c|}{ CRD } & \multicolumn{2}{l|}{ CROSSOVER } & \multicolumn{2}{l|}{ REP. LATIN SQ } \\
\hline Parameter & Estimate & $\begin{array}{l}\text { Std } \\
\text { Error }\end{array}$ & Estimate & $\begin{array}{l}\text { Std } \\
\text { Error }\end{array}$ & Estimate & $\begin{array}{l}\text { Std } \\
\text { Error }\end{array}$ \\
\hline alpha.(200) & $\begin{array}{c}0.61 \\
(0.0000)\end{array}$ & 0.0600 & $\begin{array}{c}0.46 \\
(0.0000)\end{array}$ & 0.0726 & $\begin{array}{c}0.39 \\
(0.0000)\end{array}$ & 0.0529 \\
\hline alpha.factor(1200) & $\begin{array}{c}1.61 \\
(0.0000)\end{array}$ & 0.195 & $\begin{array}{c}1.33 \\
(0.0000)\end{array}$ & 0.142 & $\begin{array}{c}1.42 \\
(0.0000)\end{array}$ & 0.0994 \\
\hline k1.(200) & $\begin{array}{c}-0.33 \\
(0.0004)\end{array}$ & 0.0935 & $\begin{array}{c}-0.44 \\
(0.0000)\end{array}$ & 0.0604 & $\begin{array}{c}-0.68 \\
(0.0000)\end{array}$ & 0.105 \\
\hline k1.factor(1200) & $\begin{array}{c}0.21 \\
(0.0031)\end{array}$ & 0.0713 & $\begin{array}{c}0.15 \\
(0.0129)\end{array}$ & 0.0618 & $\begin{array}{c}0.38 \\
(0.0003)\end{array}$ & 0.105 \\
\hline tau1.(200) & $\begin{array}{c}6.85 \\
(0.0002)\end{array}$ & 1.848 & $\begin{array}{c}8.61 \\
(0.0000)\end{array}$ & 0.683 & $\begin{array}{c}8.10 \\
(0.0000)\end{array}$ & 0.539 \\
\hline tau1.factor(1200) & $\begin{array}{c}1.63 \\
(0.1660)\end{array}$ & 1.179 & $\begin{array}{c}3.72 \\
(0.0000)\end{array}$ & 0.538 & $\begin{array}{c}3.19 \\
(0.0000)\end{array}$ & 0.653 \\
\hline delta.(200) & $\begin{array}{c}0.096 \\
(0.2268)\end{array}$ & 0.0791 & $\begin{array}{c}0.19 \\
(0.0132)\end{array}$ & 0.0747 & $\begin{array}{c}0.16 \\
(0.2296)\end{array}$ & 0.134 \\
\hline delta.factor(1200) & $\begin{array}{c}0.099 \\
(0.0296)\end{array}$ & 0.0455 & - & - & $\begin{array}{c}0.12 \\
(0.5226)\end{array}$ & 0.189 \\
\hline k2.(200) & $\begin{array}{c}-0.11 \\
(0.0001)\end{array}$ & 0.0291 & $\begin{array}{c}-0.20 \\
(0.0000)\end{array}$ & 0.0419 & $\begin{array}{c}-0.19 \\
(0.0000)\end{array}$ & 0.0322 \\
\hline k2.factor(1200) & $\begin{array}{c}0.067 \\
(0.0203)\end{array}$ & 0.0289 & $\begin{array}{c}0.014 \\
(0.8020)\end{array}$ & 0.0542 & $\begin{array}{c}0.034 \\
(0.4083)\end{array}$ & 0.0408 \\
\hline tau2.(200) & $\begin{array}{c}17.98 \\
(0.0176)\end{array}$ & 7.557 & $\begin{array}{c}26.30 \\
(0.0000)\end{array}$ & 4.951 & $\begin{array}{c}33.98 \\
(0.0000)\end{array}$ & 6.884 \\
\hline tau2.factor(1200) & $\begin{array}{c}5.33 \\
(0.0879)\end{array}$ & 3.121 & $\begin{array}{c}1.32 \\
(0.8470)\end{array}$ & 6.862 & $\begin{array}{c}-7.63 \\
(0.4291)\end{array}$ & 9.647 \\
\hline
\end{tabular}

${ }^{1}$ There is no distance effect since the crossover model did not converge when the treatment effect was specified for the recovery baseline, delta 
The maximum increase in $\mathrm{Tb}$, alpha, in 200 meter runs is significantly different from zero in all three designs. The difference in alpha for 1200 meters is significantly higher than for the 1200 meter runs. The maximum increase in $\mathrm{Tb}$ ranges from 1.79 in the crossover design to 2.23 in CRD.

The challenge rate constant, $\mathrm{k} 1$, in the 200 meter runs is significantly different from zero in all three designs and they are all negative. For the 200 meter runs, k1, is significantly larger than for the 1200 meter runs in all three designs. For the 200 meter runs, the challenge rate constant ranges from -0.68 in replicated Latin square design to -0.33 in CRD. For 1200 meters, k1, ranges from -0.30 in replicated Latin square design to -0.12 in CRD.

The challenge inflection point, tau1, for 200 meter runs is significantly different from zero in all three designs. It ranges from $6.85 \mathrm{~min}$ in CRD to $8.61 \mathrm{~min}$ in crossover design. The 1200 meter challenge inflection point is significantly larger than in the 200 meter run in the crossover design and replicated Latin square design; but not the CRD. The challenge inflection point for 1200 meters ranges from 8.49 mins in the Latin square to 12.33 mins in the crossover design.

The recovery baseline, delta, in 200 meter runs is not significantly different from zero in the CRD and replicated Latin square design. The 1200 meter is significant only in the CRD where it is $0.19 \mathrm{C}$. The crossover design model did not converge when distance was used as a treatment factor for delta. Thus, the common baseline is estimated to be 0.16 and it is significantly different from zero.

The recovery rate constant, $\mathrm{k} 2$, for 200 meters is significantly different from zero in all three designs and they are all negative. They range from -0.20 in crossover to -0.11 in CRD. The difference in $\mathrm{k} 2$, for 1200 meters is not significant in the crossover and replicated Latin square design. In CRD, the 1200 meter run is significantly greater than 200 meters run and it is -0.04 .

The time to maximum recovery rate is the recovery inflection point. For 200 meters, the recovery inflection point, tau2, is significantly different from zero in all three designs. It ranges from 17.98min in CRD to $33.89 \mathrm{~min}$ in replicated Latin square design. There is no significant change in the recovery inflection point for the 1200 meter runs.

\section{3.c Comparing Experimental Design Models}

Table 3.5 summarizes the information needed to compare how well these three designs fit the data. The CRD and crossover design are significantly different. The crossover is better than the CRD since it has a smaller AIC and BIC and a larger log-likelihood. Nevertheless, the replicated Latin square provides a significantly better fit then either of the other two designs. 
Table 3.5. Information Criteria, Log-Likelihoods, and Ratio for Comparing Experimental Design Models

\begin{tabular}{|l|l|l|l|l|l|l|l|}
\hline Design & df & AIC & BIC & logLik & Test & L.Ratio & p-value \\
\hline 1 CRD & 25 & -327.10 & -212.07 & 188.55 & & & \\
\hline 2 Crossover & 30 & -1560.33 & -1422.29 & 810.16 & 1 vs 2 & 1243.22 & $<.0001$ \\
\hline $\begin{array}{c}\text { 3 Replicated } \\
\text { Latin Square }\end{array}$ & 32 & -1719.67 & -1572.43 & 891.83 & 2 vs 3 & 163.34 & $<.0001$ \\
\cline { 5 - 8 } & & & 1 vs 3 & 1391.87 & $<.0001$ \\
\hline
\end{tabular}

\section{3.d Relative Efficiencies}

The relative efficiencies presented in Table 3.6 also help compare these three designs.

Table 3.6 Relative Efficiency for each Pair of Design Models

\begin{tabular}{|l|l|}
\hline Comparison & Relative Efficiency \\
\hline CRD vs. Crossover & 2.66 \\
\hline CRD vs. Replicated Latin Square & 3.05 \\
\hline $\begin{array}{l}\text { Crossover vs. Replicated Latin } \\
\text { Square }\end{array}$ & 1.14 \\
\hline
\end{tabular}

The relative efficiency of CRD and crossover design is 2.66. This means using the CRD requires 2.66 times as many observations as crossover design to achieve the same level of precision. Thus, the crossover design is more efficient than the CRD.

The replicated Latin square design is much more efficient than CRD (3.05). On the other hand, the replicated Latin square design is only slightly more efficient than the crossover design (1.1). This suggests a crossover design may be a viable alternative to replicated Latin squares.

\section{SUMMARY}

The Bi-logistic model provides a good fit for Tb during moving and handling of steers. The six biological parameters are important for describing the thermal challenge and recovery. In this paper, we show how to improve the model by adding random effects to account for additional sources of variation in the animals, pen, and day. While the experimental design is the traditional way to account for variation in the data, fitting a nonlinear mixed model with six parameters under extremely variable animal and environmental conditions is difficult. Introducing a treatment factor such as distance further complicates the situation. Thus, we suggest beginning with a simple model because it is easier to find starting values and obtain convergence.

However, it is important to issue the following caveat. Although it is easier to make the CRD model converge, the results in terms of treatment comparisons can be misleading. The more complex designs identify variation that cannot be overlooked. The crossover design provides a significant improvement (in fit) over the CRD. However, the experiment was designed as a replicated Latin square and that model provides the best fit.

The relative efficiency provides an interesting implication for future studies. The crossover design is almost as efficient as the replicated Latin square and may be considered a viable experimental design for future studies. 
Distance is important when moving steers in a thermally challenging environment. This study shows moving steers $1200 \mathrm{~m}$ is significantly different from moving them $200 \mathrm{~m}$. When the steers are moved $1200 \mathrm{~m}$, the maximum $\mathrm{Tb}$ is $1.4^{\circ} \mathrm{C}$ higher; the heat challenge rate constant is smaller (indicating temperature is rising faster) and the challenge inflection point (time to maximum rate of challenge) occurs later in the run. Thus, the steers are more challenged when moved longer distances. These results suggest moving and handling cattle should be carefully managed during the summer to avoid heat stress and even death of the animals.

\section{REFERENCES}

Aitha, N, A. M. Parkhurst, C. N. Lee and P. E. Hillman, 2008. Nonlinear mixed models to evaluate effects of environment conditions, hair coat, and anchor length on body temperature during afternoon milking of Holstein. Proceedings of Twentieth Annual Kansas State University Conference on Applied Statistics in Agriculture Proceedings. 206-217 http://www.kstate.edu/stats/agstat.conference/2008

Kerek, M.,A.M.Parkhurst, and T. L. Mader, 2003. Using the bi-logistic model to estimate body temperature in feedlot cattle, Proceedings of Fifteenth Annual Kansas State University Conference on Applied Statistics in Agriculture Proceedings. 206-217

http://www.k-state.edu/stats/agstat.conference/2003

Parkhurst, A. M. and T. L. Mader. 2000. Using nonlinear growth curves to estimate heat stress in processing feedlot cattle. Proceedings of Twelfth Annual Kansas State University Conference on Applied Statistics in Agriculture Proceedings. 103-118

http://www.k-state.edu/stats/agstat.conference/2000

Pinheiro, Jose, Douglas Bates, Saikat DebRoy, Deepayan Sarkar and the R Core team 2008. nlme: Linear and Nonlinear Mixed Effects Models. R package version 3.1-89.

R Development Core Team 2008. R: A language and environment for statistical computing. R Foundation for Statistical Computing,Vienna, Austria. ISBN 3-900051-07-0, URL http://www.R-project.org.

Zhou M., A.M. Parkhurst, R.A.Eigenberg, J.Nienaber, L.Hahn, 2006. Evaluating nonlinear crossed random effects models for comparing temperature of eating pigs under different thermal environments. Proceedings of Eighteenth Annual Kansas State University Conference on Applied Statistics in Agriculture Proceedings. 180-200

http://www.k-state.edu/stats/agstat.conference/2006

Zhou M., A.M. Parkhurst, B.C. Pollard, R.J. Collier, 2007. Using a nonlinear crossed random effects model with three-way treatment structure for describing circadian patterns of serum prolactin concentrations in heat stressed Holsteins. Proceedings of Nineteenth Annual Kansas State University Conference on Applied Statistics in Agriculture Proceedings. 126-143 http://www.k-state.edu/stats/agstat.conference/2007 